\title{
DARPP-32: from neurotransmission to cancer
}

\author{
Abbes Belkhiri ${ }^{1}$, Shoumin Zhu ${ }^{1}$ and Wael El-Rifai ${ }^{1,2}$ \\ ${ }^{1}$ Department of Surgery, Cancer Biology, and Vanderbilt-Ingram Cancer Center, Vanderbilt University Medical Center, \\ Nashville, TN, USA \\ 2 Department of Veterans Affairs, Tennessee Valley Healthcare System, Nashville, TN, USA \\ Correspondence to: Wael El-Rifai, email: wael.el-rifai@vanderbilt.edu \\ Keywords: DARPP-32, t-DARPP, PPPIR1B, neurotransmission, cancer \\ Received: November 05, $2015 \quad$ Accepted: January 29, 2016 \\ Published: February 08, 2016
}

\section{ABSTRACT}

Dopamine and cAMP-regulated phosphoprotein Mr 32,000 (DARPP-32), also known as phosphoprotein phosphatase-1 regulatory subunit 1B (PPP1R1B), was initially discovered as a substrate of dopamine-activated protein kinase $A$ (PKA) in the neostriatum in the brain. While phosphorylation at Thr-34 by PKA converts DARPP-32 into a potent inhibitor of protein phosphatase 1 (PP1), phosphorylation at Thr-75 transforms DARPP-32 into an inhibitor of PKA. Through regulation of DARPP-32 phosphorylation and modulation of protein phosphatase and kinase activities, DARPP-32 plays a critical role in mediating the biochemical, electrophysiological, and behavioral effects controlled by dopamine and other neurotransmitters in response to drugs of abuse and psychostimulants. Altered expression of DARPP-32 and its truncated isoform (t-DARPP), specifically in the prefrontal cortex, has been associated with schizophrenia and bipolar disorder. Moreover, cleavage of DARPP-32 by calpain has been implicated in Alzheimer's disease. Amplification of the genomic locus of DARPP-32 at 17q12 has been described in several cancers. DARPP-32 and t-DARPP are frequently overexpressed at the mRNA and protein levels in adenocarcinomas of the breast, prostate, colon, and stomach. Several studies demonstrated the pro-survival, pro-invasion, and pro-angiogenic functions of DARPP-32 in cancer. Overexpression of DARPP-32 and t-DARPP also promotes chemotherapeutic drug resistance and cell proliferation in gastric and breast cancers through regulation of pro-oncogenic signal transduction pathways. The expansion of DARPP-32 research from neurotransmission to cancer underscores the broad scope and implication of this protein in disparate human diseases.

\section{INTRODUCTION}

In a study to characterize the mode of action of neurotransmitters in different brain regions, Walaas and colleagues [1] investigated proteins phosphorylated by calcium- and cyclic-AMP (cAMP)-activated protein kinases. Notably, a $32-\mathrm{kDa}$ protein was found abundantly in spiny neurones of the neostriatum. As the phosphorylation of this protein was regulated by dopamine and cAMP, it was named DARPP-32 (dopamine and cAMP-regulated phosphoprotein Mr 32,000) [2]. In addition to the brain, DARPP-32 is expressed in adrenal medulla, kidney, and parathyroid cells [3-5]. Because it was shown to inhibit protein phosphatase-1 (PP1) following phosphorylation of Thr-34 by protein kinase A (PKA) [6], DARPP-32 is also known as phosphoprotein phosphatase-1 regulatory subunit 1B (PPP1R1B). DARPP-32 has been shown to play a key role in mediating the biochemical, electrophysiological, and behavioral effects of dopamine on dopaminoceptive neurons (for reviews, see [7-9]). DARPP-32 has also been implicated in mediating the actions of other neurotransmitter systems such as serotonin and glutamate, in response to a variety of drugs of abuse (for reviews, see [10,11]). El-Rifai and colleagues discovered frequent amplification of $17 q 12$, the locus of DARPP-32, in gastric and esophageal adenocarcinomas $[12,13]$. Subsequently, this group reported that DARPP-32 and its truncated isoform; they cloned and named t-DARPP, are amplified and overexpressed in gastric cancer [14]. This first report led to a series of studies on the role of DARPP-32 and t-DARPP in different biological aspects of gastric cancer and other 
malignancies. In this review, we briefly outline the role of DARPP-32 in neurotransmission and its recent implication in central nervous system (CNS) disorders, and elaborate on the novel biological functions of DARPP-32 and t-DARPP in human carcinogenesis.

\section{MODULATION OF DARPP-32 IN NEUROTRANSMISSION AND CNS DISORDERS}

Since its discovery three decades ago, DARPP-32 has been shown in a large body of work as a central signaling molecule activated by a diverse array of neurotransmitters such as dopamine, glutamate, serotonin, adenosine, and gamma-aminobutyric acid (GABA) $[9,15,16]$. In response to drugs of abuse and psychostimulants, these neurotransmitters regulate the phosphorylation state of DARPP-32, which converts it to an inhibitor of either a protein phosphatase (PP1) or a protein kinase (PKA). The state of phosphorylation of DARPP-32 is regulated by tonic activation of dopamine $\mathrm{D}_{1}$ and $\mathrm{D}_{2}$ receptors, and adenosine $\mathrm{A}_{2 \mathrm{~A}}$ receptor [17]. DARPP-32 may be phosphorylated at Thr-34 by PKA in distinct subpopulations of medium spiny neurons that express either $\mathrm{D}_{1}$ or $\mathrm{A}_{2 \mathrm{~A}}$ receptors $[18,19]$. Like dopamine, adenosine acts on $\mathrm{A}_{2 \mathrm{~A}}$ receptor, using cAMP as a mediator in the process, activating PKA and increasing DARPP-32 phosphorylation at Thr-34 [20]. The $\mathrm{D}_{2}$ receptor exists as two different isoforms generated by alternative splicing: the long (D2L) and the short (D2S) [21]. The D2S receptor specifically regulates the state of phosphorylation and activity of tyrosine hydroxylase (TH) in nigrostriatal presynaptic terminals, whereas the $\mathrm{D} 2 \mathrm{~L}$ receptor is mainly involved in the regulation of DARPP-32 phosphorylation in postsynaptic striatal medium spiny neurons [22]. Activation of the $\mathrm{D}_{2}$ receptor decreases DARPP-32 phosphorylation by two different mechanisms. First, in medium spiny neurons that co-express $\mathrm{D}_{1}$ and $\mathrm{D}_{2}$ classes of dopamine receptors, activation of $\mathrm{D}_{2}$ receptors decreases cAMP levels. When $\mathrm{D}_{2}$ receptors are co-expressed with adenosine $\mathrm{A}_{2 \mathrm{~A}}$ receptor, it can result in a decrease of cAMP levels, decreasing the activity of both PKA and in phosphorylation of DARPP-32 at Thr-34. Second, activation of $\mathrm{D}_{2}$ receptor leads to an increase in $\mathrm{Ca}^{2+}$ levels and increased activity of protein phosphatase $2 \mathrm{~B}$ (PP-2B), resulting in an increase in dephosphorylation of DARPP-32 at Thr-34 [23]. In the intact striatum, a blockade of tonic dopamine $\mathrm{D}_{2}$ receptor activation increases DARPP-32 phosphorylation at Thr-34 and can be counteracted by blocking either adenosine $\mathrm{A}_{2 \mathrm{~A}}$ or dopamine $\mathrm{D}_{1}$ receptors [17].

The phosphorylation of DARPP-32 at Thr-34 converts it into a potent inhibitor of PP1 [6, 24, 25]. PP1 is involved in regulating the activity of a large number of phosphoproteins, including voltage-dependent sodium and calcium channels, the electrogenic pump $\mathrm{Na}^{+}, \mathrm{K}^{+}-$ ATPase, and neurotransmitter receptors [26]. DARPP-32mediated inhibition of PP1 increases the phosphorylation of neurotransmitter receptors and ion channels crucial for synaptic function and plasticity [9]. Bibb and colleagues reported that DARPP-32 was converted into an inhibitor of PKA when phosphorylated at Thr-75 by cyclin-dependent kinase 5 (Cdk5) [27]. Decreasing phospho-Thr-75 DARPP-32 in striatal slices, either by a Cdk5-specific inhibitor or by using genetically altered mice, increases dopamine-induced phosphorylation of PKA substrates and augments peak voltage-gated calcium currents [28, 29]. This suggests that, depending on which particular amino acid residue is phosphorylated, DARPP-32 can function as either a kinase or a phosphatase inhibitor. This unique dual action appears to be especially important in regulating the efficacy of dopaminergic neurotransmission [8, 27] (Figure 1).

Casein kinase 1 (CK1) can phosphorylate

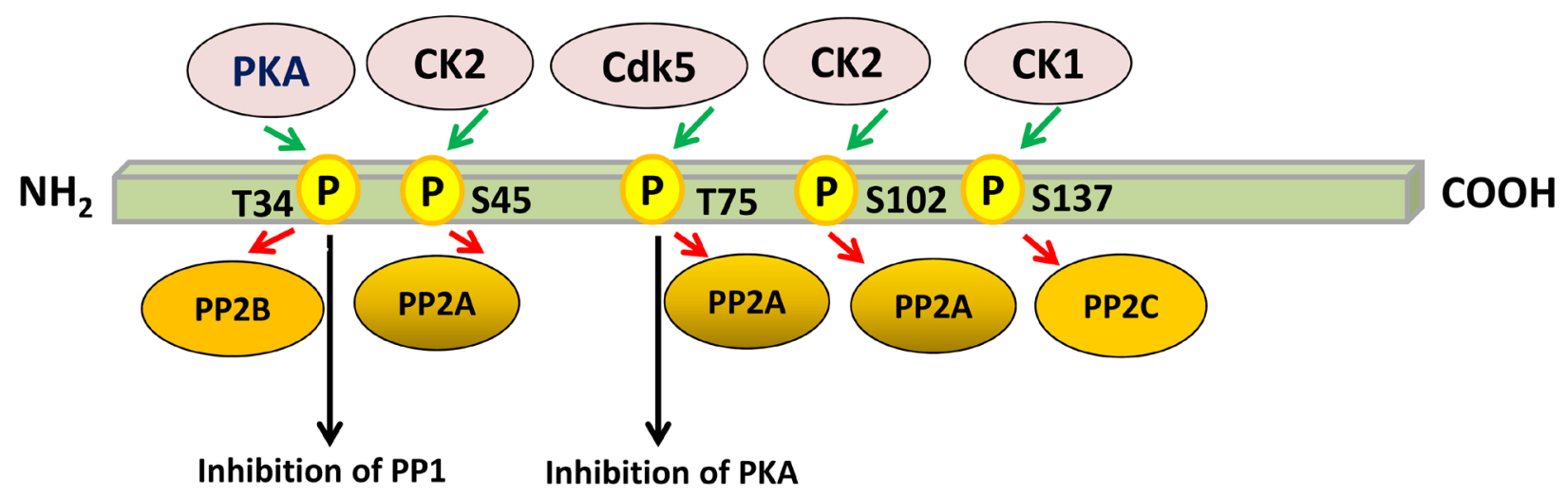

Figure 1: Regulation of PP1 and PKA by multisite phosphorylation of DARPP-32. Phosphorylation of DARPP-32 by protein kinases is indicated by green arrows, whereas dephosphorylation of DARPP-32 by protein phosphatases is depicted by red arrows. Phosphorylation of DARPP-32 at Thr-34 by PKA converts it into a potent inhibitor of PP1. However, phosphorylation of DARPP-32 at Thr- 75 by CDK 5 transforms it into an inhibitor of PKA. Dephosphorylation of Ser-137 by PP2C facilitates dephosphorylation of Thr-34 by PP2B, thereby removing the PKA-induced inhibition of PP1. Phosphorylation of Ser-45 and Ser-102 has no effect on the potency of DARPP-32 as an inhibitor of PP1. The scheme is based on [29]. 
DARPP-32 at Ser-137, which has an important regulatory role because it inhibits the dephosphorylation of Thr-34 by calcineurin. This inhibition takes place only when phospho-Ser-137 and phospho-Thr-34 are present on the same DARPP-32 molecule [30]. Phosphorylation of DARPP-32 by CK1, which is highly active in striatonigral neurons, does not alter its ability to inhibit PP1 [30]. DARPP-32 phosphorylated by CK1 is a substrate for protein phosphatases $2 \mathrm{~A}$ and $2 \mathrm{C}$. However, in substantia nigra slices, dephosphorylation of Ser-137 was markedly sensitive to decreased temperature, and not significantly affected by the presence of okadaic acid under conditions where dephosphorylation of Thr-34 by protein phosphatase $2 \mathrm{~A}$ was inhibited. This suggests that, in neurons, phospho-Ser-137 DARPP-32 is dephosphorylated by protein phosphatase $2 \mathrm{C}$, but not $2 \mathrm{~A}$. Thus, DARPP-32 appears to be a component of a regulatory cascade of phosphatases in which dephosphorylation of Ser-137 by protein phosphatase $2 \mathrm{C}$ facilitates dephosphorylation of Thr-34 by calcineurin, removing the cAMP-induced inhibition of PP1 [31]. DARPP-32 is also phosphorylated on Ser-45 and Ser-102 by casein kinase 2 (CK2) [32]. Phosphorylation by CK2 has no effect on the potency of DARPP-32 as an inhibitor of PP1, which strictly depends on phosphorylation of Thr-34 by cAMP-dependent protein kinase [33] (Figure 1).

Following the discovery of t-DARPP by El-Rifai's group in cancer [14], post-mortem studies on human brains suggest that the expression of t-DARPP transcript is altered in patients with schizophrenia and bipolar disorder specifically in the prefrontal cortex, and the higher t-DARPP expression was associated with worse cognitive performance [34]. Another study indicated a selective reduction in DARPP-32 expression in the prefrontal cortex in patients with schizophrenia, suggesting that altered expression of DARPP-32 may be associated with the development of the disease [35]. Cho and colleagues investigated the role of DARPP-32 in Alzheimer's disease (AD), and found that DARPP-32 is cleaved at Thr-153 by calpain, which reduces the phosphorylation of cAMPresponse element-binding protein (CREB), a target of PP1 and important for cognitive function. The cleavage of DARPP-32 induces loss of its inhibitory function on PP1. The data suggest a novel mechanism by which cleavage of DARPP-32 leads to dysregulation of CREB signaling in $\mathrm{AD}$ [36].

\section{IDENTIFICATION AND IMPLICATION OF DARPP-32 IN CANCER}

Earlier studies using comparative genomic hybridization have pointed out to frequent amplification at the $17 q 12$ locus in several malignancies including gastric and esophageal cancers $[12,13,37]$. A systematic analysis of copy number and expression levels of the $17 \mathrm{q}$ ampliconspecific genes revealed an increase in copy numbers of
18 genes such as $E R B B 2, T O P 2 A, G R B 7$, and expression sequence tag (EST AA552509) in gastric cancers [38]. ElRifai and colleagues demonstrated that EST AA552509 was frequently amplified and consistently overexpressed at $17 \mathrm{q}$ in gastric cancers, and they were first to report that DARPP-32 is the target gene for overexpression of EST AA552509 [14]. Cloning and sequence assembly analyses indicated that EST AA552509 corresponded to the 3'-untranslated region of the DARPP-32 gene. Further analyses of the nearby ESTs, two ESTs (BF725600 and BF724182) were found to overlap with the 5' end of DARPP-32 exon 1. An additional 467 bp untranslated mRNA sequence of DARPP-32 was identified upstream of the previously known translation start site in exon 1 . Additional cloning and sequencing verified the fulllength DARPP-32 cDNA sequence (AF464196). A novel transcriptional splice variant of DARPP-32 was cloned from gastric cancer tissues (AY070271) and termed truncated isoform of DARPP (t-DARPP) [14]. The novel molecule, t-DARPP, has a unique alternative first exon located within intron 1 of DARPP-32. While DARPP-32 encodes 204 amino acids protein containing four phosphorylation sites (Thr-34, Thr-75, Ser-102, and Ser-137), t-DARPP encodes 168 amino acids protein lacking the Thr-34 phosphorylation site related to PP1 inhibition but maintains the other three phosphorylation sites (Figure 2). Concomitant overexpression of DARPP-32 and t-DARPP was demonstrated in 68\% of gastric cancers [39]. In addition, several studies indicated frequent overexpression of DARPP-32 and t-DARPP in adenocarcinomas of the breast, prostate, and colon [4043]. These findings suggest that DARPP-32 proteins could be implicated in critical steps in the carcinogenesis cascade. Further studies were prompted to investigate the functional role of DARPP-32 and t-DARPP primarily in gastric cancer and other types of malignancies.

\section{DARPP-32 in tumorigenesis}

A few studies have attempted to examine the role of DARPP-32 in the multistep cascade of gastric carcinogenesis. A study using immunohistochemistry analysis on 533 human gastric cancer tissues indicated that DARPP-32 is overexpressed in early stages of gastric tumorigenesis, suggesting that this molecular event may participate in the transition to intestinal metaplasia and in the progression to adenocarcinoma [44]. Based on the concept that fusion genes, which result from chromosomal rearrangements or abnormal transcription, act as potent oncogenes in many types of cancer $[45,46]$, Yun and colleagues [47] identified novel gene fusions in human gastric cancer cells and tissues. Notably, the presence of DARPP-32-STARD3 fusion transcript, which appears to be generated by RNA processing without chromosomal rearrangement, was exclusively detected in a subset of 
gastric cancers $(21.3 \%)$ but not in adjacent matched normal gastric tissues. Overexpression of DARPP-32-STARD3 significantly increases gastric cancer cell proliferation in vitro and gastric tumor xenograft growth in nude mice. These effects are mediated by activation of the PI3K/ AKT signaling pathway [47]. These findings strongly suggest that DARPP-32-STARD3 fusion transcript promotes tumorigenesis in gastric cancer. Another study included an investigation on the role of DARPP-32 in gastric tumorigenesis through regulation of CD44 splicing [48]. The findings indicated that DARPP-32 positively regulates the overall splicing activity, and specifically promotes the expression of the splice isoform, CD44E, in gastric cancer cells. Mechanistic studies revealed that DARPP-32 associates with SRp20 splicing factor in a protein complex, thereby stabilizing SRp20 protein and increasing its expression. Modulation of DARPP-32 expression indicated that DARPP-32 regulates the SRp20-dependent CD44E splicing and gastric tumor growth in a xenograft mouse model. Notably, the study indicated frequent overexpression of DARPP-32, SRp20 and CD44E proteins in human gastric primary tumors, further establishing an important role for DARPP-32 in gastric tumorigenesis [48]. In a recent report, the role of DARPP-32 and t-DARPP in breast tumorigenesis was investigated in a mouse mammary tumor model [49]. In contrast to DARPP-32, which was expressed in normal mouse mammary tissue and in some tumors, t-DARPP was exclusively expressed in tumors at equal or higher levels than DARPP-32, suggesting that t-DARPP plays a predominant role in breast tumorigenesis. Notably, knockout of the DARPP-32 gene in MMTV (mouse mammary tumor virus promoter)-PyMT (polyoma middle $\mathrm{T}$ oncogene) transgenic mammary tumor mice reduced tumor growth, thereby demonstrating the important role of DARPP-32 proteins in breast tumorigenesis [49].

\section{DARPP-32 proteins in cancer cell survival and drug resistance}

An initial report demonstrated that DARPP-32 proteins could protect cancer cells against drug-induced apoptosis. This was particularly evident in studies where both DARPP-32 and t-DARPP protected gastric cancer cells against camptothecin, sodium butyrate, and ceramide treatments in a p53-independent mechanism [39]. Expression of DARPP-32 and t-DARPP preserved the mitochondrial transmembrane potential in response to camptothecin, and this was associated with increased levels of $\mathrm{Bcl} 2$ protein [39]. Notably, the pro-survival function of both DARPP-32 and t-DARPP depends on Thr-75 phosphorylation residue [39]. A subsequent study indicated that the pro-survival function of t-DARPP involves increased AKT kinase activity and high levels of phospho-AKT (Ser-473) and phospho-GSK3 $\beta$ (Ser9) proteins in gastric cancer cells [50]. In addition, the study demonstrated that t-DARPP activates CREB and ATF-1 transcription factors, which bind to a CREB response element (CRE) on $\mathrm{Bcl} 2$ promoter, in an AKTdependent mechanism, leading to up-regulation of Bcl2 expression and increased cell survival in response to

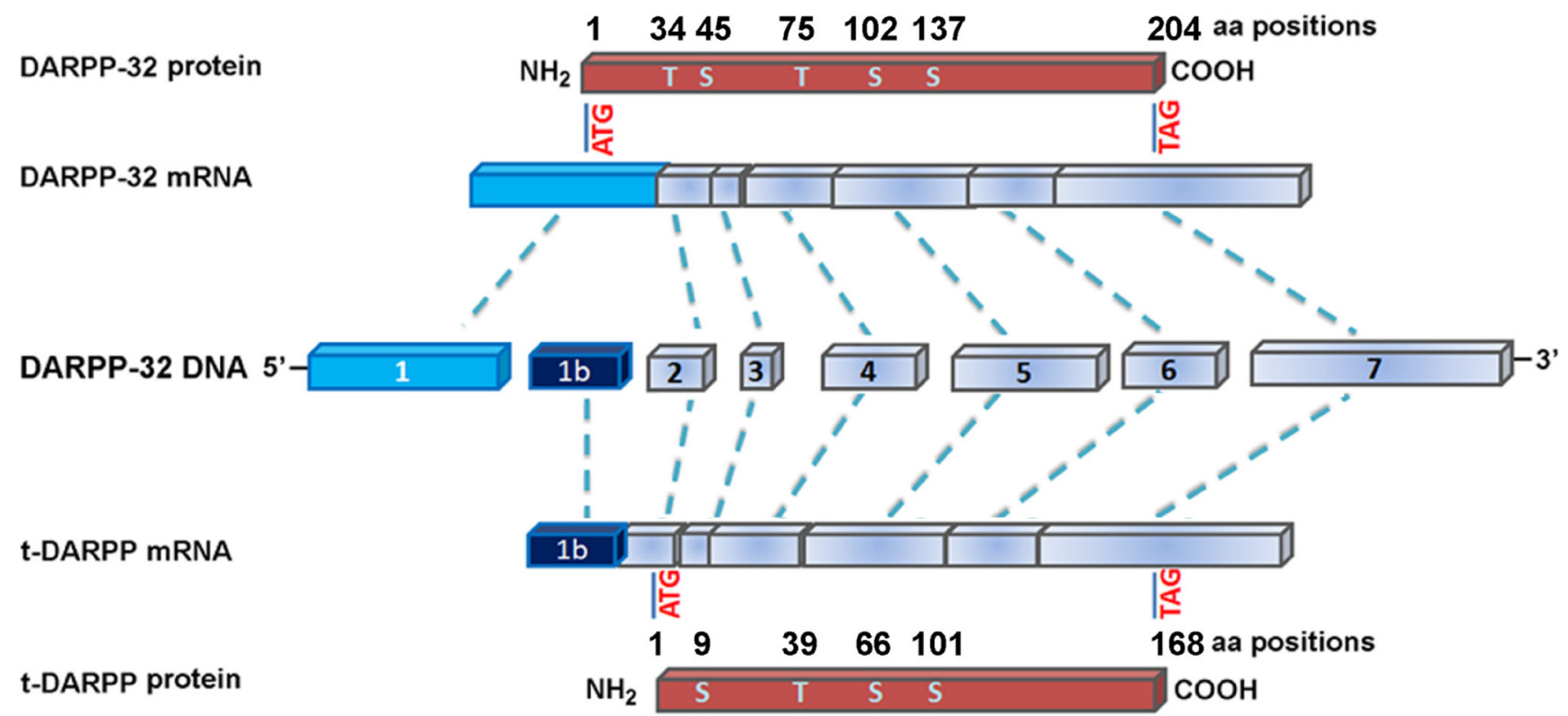

Figure 2: Genomic structure of DARPP-32 and its truncated isoform, t-DARPP. DARPP-32 and t-DARPP share identical sequence from exon 2 to the 3' end. Of note, exon 1 of t-DARPP is spliced from the intron 1 of DARPP-32. DARPP-32 (1,983 bp) encodes 204 amino acids, whereas t-DARPP (1,502 bp) encodes 168-amino-acids protein. The major phosphorylation sites on the proteins are indicated by $\mathrm{T}$, threonine, and S, serine. DARPP-32 contains five phosphorylation sites at T34, S45, T75, S102, and S137, whereas t-DARPP lacks the T34 phosphorylation site of DARPP-32. The scheme is based on [39]. 
ceramide [50]. A recent report indicated that DARPP-32 binds $\mathrm{Bcl} 2$ and calcineurin $(\mathrm{CaN})$ in a complex with inositol 1,4,5-triphosphate receptor (InsP3R, a ubiquitous intracellular $\mathrm{Ca} 2+$ channel), which induces a negative feedback loop that reduces $\mathrm{Ca}+2$ release and apoptosis by decreasing phosphorylation of InsP3R in chronic lymphocytic leukemia cells [51]. The study concluded that this mechanism involving DARPP-32 might be exploited by Bcl2-overexpressing cancer cells to suppress apoptosis. On the other hand, one study found that DARPP-32 reversed the multidrug resistance (MDR) of Adriamycin (ADR)-resistant gastric adenocarcinoma cells (SGC7901/ ADR), as these cells were significantly sensitized to vincristine, ADR, 5-fluorouacil, and cisplatin in response to DARPP-32 up-regulation [52]. Based on a single gastric cancer cell model, these controversial findings may suggest cell type specificity.

In a study, the role of DARPP-32 in mediating resistance to gefitinib, a small tyrosine kinase inhibitor specific for EGFR, was investigated in gastric cancer. The data demonstrated that DARPP-32 expression blocks gefitinib-induced apoptosis in gastric cancer cells. This is accompanied with activation of AKT, increased stability of the EGFR protein, and the colocalization of DARPP-32 with EGFR in a complex with ERBB3 on the cell membrane [53]. Mechanistic investigations concluded that DARPP-32 promotion of EGFR/ERBB3 protein interaction leads to EGFR phosphorylation and activation of PI3K-AKT signaling, thereby increasing cell survival and gefitinib resistance in gastric cancer cells [53]. In a report, DARPP-32 was implicated in mediating resistance to TRAIL (TNF-related apoptosisinducing ligand) in gastric cancer. The data indicated that DARPP-32 expression suppresses TRAIL-induced apoptosis, accompanied with reduced cytochrome c release and activation of caspase- $8,-9$, and -3 in gastric cancer cells [54]. Further investigations indicated that DARPP-32 induces the expression of the pro-survival Bcl-xL protein through activation of Src/STAT3 signaling. In addition, DARPP-32 has been shown to prevent the TRAIL-induced caspase-dependent cleavage of NF$\kappa B p 65$, thereby maintaining the activity of NF- $\kappa B$ activity and the expression of its target, the pro-survival FLIPs (FLICE-inhibitory protein). Together, the findings suggest that DARPP-32 promotes TRAIL resistance whereby it suppresses the intrinsic and extrinsic apoptosis pathways through regulation of Bcl-xL and FLIPs, respectively [54].

In a study, the role of t-DARPP in resistance to trastuzumab (Herceptin), a monoclonal antibody against ERBB2 receptor, was investigated in breast cancer [55]. The data indicated that t-DARPP expression was up-regulated in trastuzumab-resistant ERBB2-positive breast cancer cells. Genetic knockdown of t-DARPP sensitized these resistant cells to trastuzumab as indicated by increased activation of caspase- 3 and apoptosis. Mechanistic studies demonstrated that t-DARPP was associated with the chaperone heat shock protein 90 (HSP90) and ERBB2 in a protein complex, suggesting that t-DARPP mediates trastuzumab resistance through regulation of ERBB2 receptor in trastuzumab-resistant breast cancer cells [55]. A subsequent study confirmed that t-DARPP overexpression was associated with AKT activation and trastuzumab resistance in breast cancer cells [56]. The findings indicated that t-DARPP overexpression and Thr-75 t-DARPP residue are essential for AKT activation and trastuzumab resistance in these cells. In addition, the study showed that DARPP-32 promotes resistance to trastuzumab, and overexpression of DARPP32 is associated with poor prognosis in breast cancer [56]. However, another study indicated that t-DARPP, but not DARPP-32, was overexpressed in a trastuzumab-resistant breast cancer cells, and demonstrated that t-DARPP overexpression was sufficient to confer resistance to trastuzumab and suppress trastuzumabmediated dephosphorylation of AKT in sensitive breast cancer cells [42]. A recent study has shown that t-DARPP overexpression in HER2-positive breast cancer confers a survival advantage to cancer cells in response to lapatinib by suppressing lapatinib-induced BIM accumulation [40]. A related report indicated that t-DARPP promotes trastuzumab resistance through regulation of ERBB2 receptor in esophageal adenocarcinoma [57]. In fact, t-DARPP was shown to counteract trastuzumab-induced apoptosis, inhibit activation of caspase-3, and suppress trastuzumab-induced dephosphorylation of ERBB2 and AKT proteins. However, knockdown of endogenous t-DARPP reversed these effects and sensitized cells to trastuzumab. The findings from this study also indicated that t-DARPP associates with ERBB2, thus increasing its protein stability and interfering with the binding of trastuzumab with the ERBB2 receptor in esophageal adenocarcinoma cells [57].

\section{DARPP-32 proteins in cancer cell growth and proliferation}

A study demonstrated mRNA overexpression of t-DARPP in $36 \%$ of breast cancers, predominantly observed in invasive ductal and intraductal carcinomas, as opposed to absent to very low t-DARPP expression in normal mammary tissue. Similarly, protein overexpression of DARPP-32/t-DARPP was observed in $35.5 \%$ of primary breast tumors, including invasive ductal carcinomas (43.7\%) [41]. In vitro studies demonstrated that the overexpression of t-DARPP promotes cell proliferation associated with induction of phosphorylation of AKT (Ser-473) and its target GSK-3 $\beta$ (Ser-9) in breast cancer cells. The knockdown of endogenous t-DARPP or inhibition of PI3K reversed these effects, suggesting that the pro-growth function of t-DARPP depends on PI3K in breast cancer [41]. In a related study, the role of t-DARPP 
in regulating $\beta$-catenin signaling and proliferation was examined in upper gastrointestinal cancer cells [58]. The data indicated that the expression of t-DARPP increased cell proliferation and led to activation of $\beta$-catenin through phosphorylation of AKT (Ser-473) and GSK-3 $\beta$ (Ser-9) in a PI3K-dependent mechanism [58].

\section{DARPP-32 in cancer cell migration, invasion, and angiogenesis}

A report indicated that overexpression of DARPP-32 in breast cancer cells that express DDR1, a receptor tyrosine kinase, significantly impaired cell migration. However, DARPP-32 expression in DDR1deficient cells had no effect on migration [59]. It was demonstrated that DDR1 associates with DARPP-32 in a protein complex, which regulates migration, and the phosphorylation of Thr-34 residue is required for DARPP-32 function to impair migration of breast cancer cells. This was further supported using PKA inhibitors that abrogated the anti-migratory effect of DARPP-32 [59]. A related study investigated the role of the Wnt-5a signaling pathway in regulating DARPP-32-dependent inhibition of migration in breast cancer cells [60]. The study indicated that recombinant Wnt-5a induced cAMP elevation, leading to activation of PKA, phosphorylation of Thr-34-DARPP-32, and inhibition of cell migration. Further studies demonstrated that the inhibition of PP1 by phospho-Thr-34-DARPP-32 potentiated the Wnt5a-mediated phosphorylation of CREB, a known PP1 substrate. Additionally, inhibition of the Wnt-5a-DARPP32-CREB pathway, by expression of dominant negative CREB, reduced the anti-migratory effect of DARPP-32 in response to Wnt-5a [60]. On the other hand, another study showed that DARPP-32 overexpression promotes gastric cancer cell invasion [61]. The pro-invasive function of DARPP-32 was associated with an increase in the expression of the membrane-type 1 matrix metalloproteinase (MT1-MMP) and CXC-chemokine receptor 4 (CXCR4) proteins. Consistent with the role of MT1-MMP in activating MMPs, a substantial increase in MMP-2 activity was detected in DARPP-32-expressing cells. Inhibition or knockdown of CXCR4 suppressed DARPP-32-induced cell invasion. Further mechanistic studies showed that DARPP-32 and CXCR4 coexist in the same protein complex, leading to increased stability of CXCR4 protein because of reduced ubiquitination, following treatment with its ligand, CXCL12. These findings demonstrate that DARPP-32 enhances gastric cancer cell invasion by regulating CXCR4-mediated activation of the MT1-MMP/MMP2 pathway [61]. A

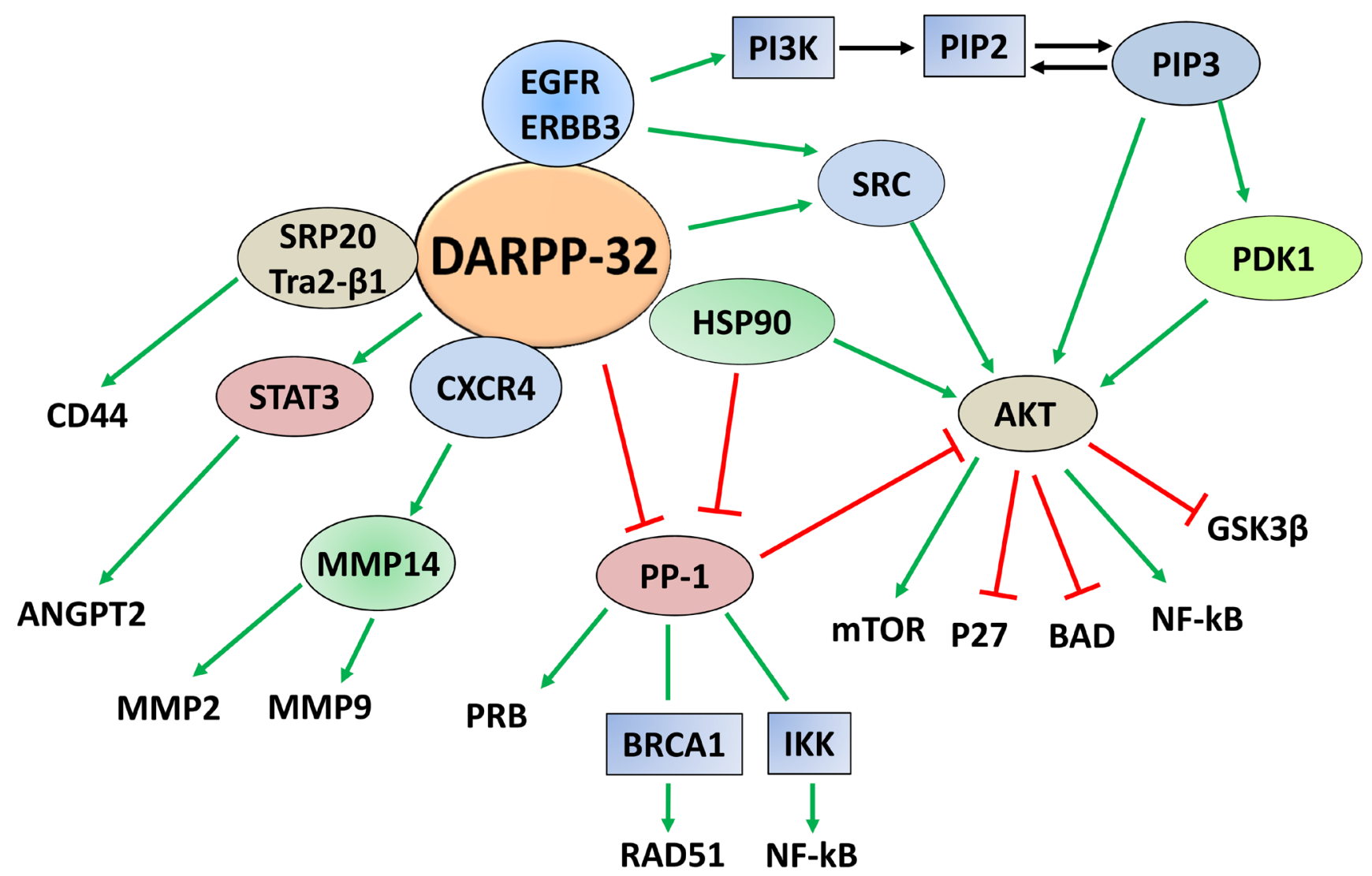

Figure 3: DARPP-32-regulated cancer signaling pathways. Based on the published data, DARPP-32 constitutes a signaling hub that regulates multiple pathways important for carcinogenesis and tumor progression. Activation is depicted by green arrows and negative regulation is indicated by red T-lines. 
prospective pilot study, using clinical and histopathological data of 100 patients with colorectal cancer between 1994 and 1997, revealed that DARPP-32 was highly expressed in patients with distant metastases, relative to patients without distant metastases. Further analysis indicated that DARPP-32 expression in primary colorectal tumors was a significant predictor of distant metastases, and could be a potential marker of poor prognosis [62]. A recent report indicated that DARPP-32 promotes angiogenesis through regulation of angiopoietin 2 (ANGPT2) in gastric cancer [63]. In fact, DARPP-32 or t-DARPP positively regulates mRNA and protein expression and secretion of ANGPT2 in gastric cancer cells, thereby inducing angiogenesis. Further mechanistic investigations confirmed the role of ANGPT2 in mediating DARPP-32-induced angiogenesis, and revealed that DARPP-32 proteins regulate ANGPT2 expression through activation of STAT3. Notably, DARPP-32 induces ANGPT2 expression in human gastric tumor epithelial cells, but not in tumor associated vascular endothelial cells [63]. A schematic diagram summarizing DARPP-32-regulated signaling pathways involved in tumorigenesis is shown in Figure 3.

\section{CONCLUSIONS AND PERSPECTIVES}

A large body of research over the last three decades has established DARPP-32 as a major integrator of neurotransmission by dopamine and other neurotransmitters in response to drugs of abuse or psychostimulants. Additionally, several recent reports implicated DARPP-32 and t-DARPP proteins in CNS disorders and neurodegenerative diseases. The role of DARPP-32 proteins in tumorigenesis and drug resistance has been recently recognized and is a growing field of investigations that continue to unveil new functions of DARPP-32 (Figure 4). Collectively, the findings from the published reports strongly suggest that DARPP-32 proteins have potential as a biomarker of drug resistance or a therapeutic target in gastric cancer and other malignancies. In the era of personalized cancer medicine, it is imperative that future investigations and efforts align together for the development and testing specific pharmacological inhibitors that target DARPP-32 and its signaling pathways in preclinical and clinical settings.

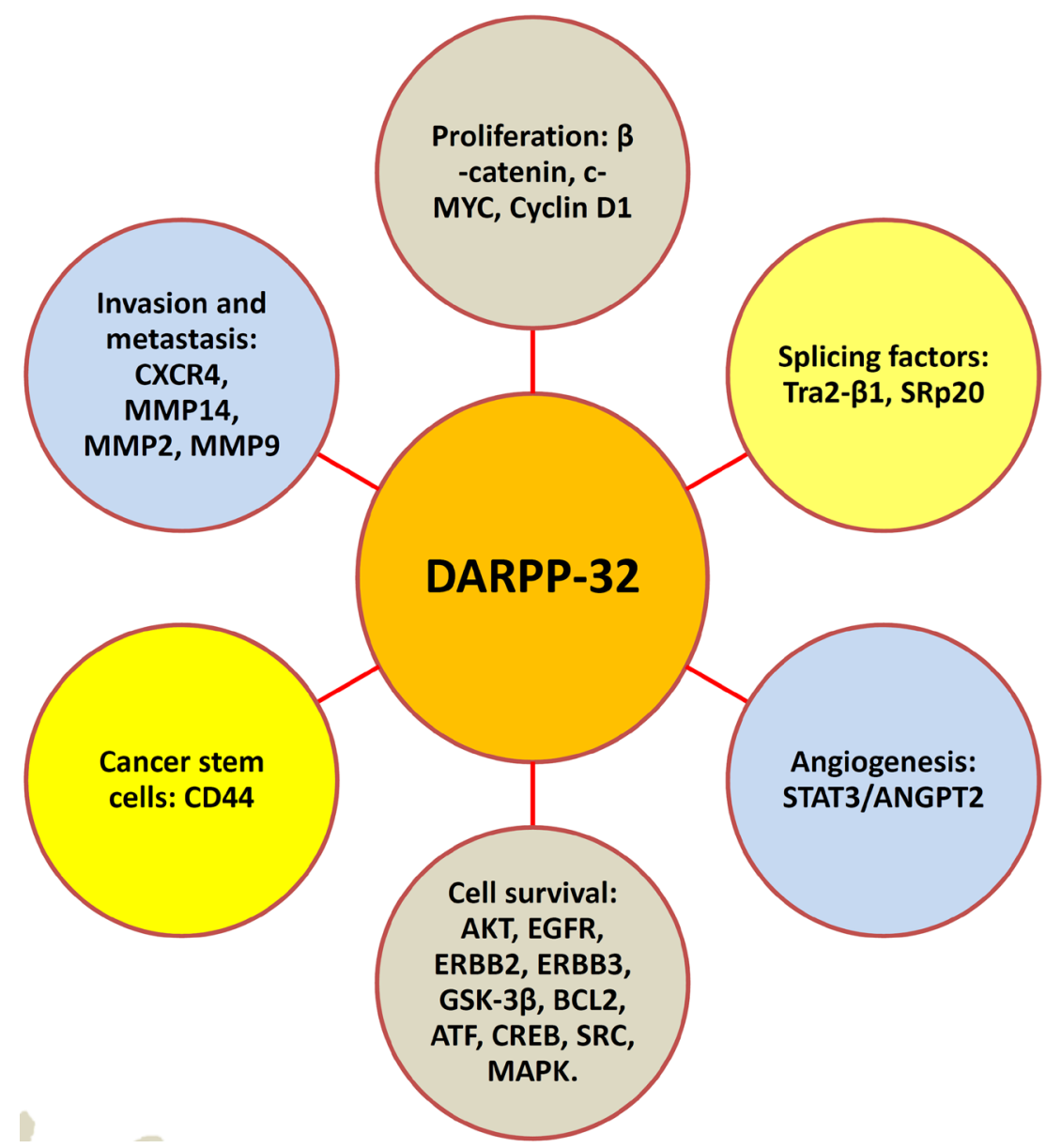

Figure 4: DARPP-32 regulates major hallmarks of tumorigenesis. Accumulating published reports strongly suggest that DARPP-32 promotes cancer cell proliferation, survival, invasion and metastasis, and angiogenesis. Each DARPP-32-mediated function involves regulation of corresponding signaling pathways depicted in the schematic diagram. 


\section{ACKNOWLEDGMENTS}

This review was supported by grants from the National Institutes of Health; R01CA93999 and the Department of Veterans Affairs. The funders had no role in study design, data collection and analysis, decision to publish, or preparation of the manuscript. The contents of this work are solely the responsibility of the authors and do not necessarily represent the official views of the National Cancer Institute, Department of Veterans Affairs, or Vanderbilt University.

\section{CONFLICTS OF INTEREST}

There is no conflict of interest.

\section{REFERENCES}

1. Walaas SI, Nairn AC and Greengard P. Regional distribution of calcium- and cyclic adenosine 3':5'-monophosphateregulated protein phosphorylation systems in mammalian brain. I. Particulate systems. J Neurosci. 1983a; 3:291-301.

2. Walaas SI, Aswad DW and Greengard P. A dopamineand cyclic AMP-regulated phosphoprotein enriched in dopamine-innervated brain regions. Nature. 1983b; 301:6971.

3. Ouimet CC, Miller PE, Hemmings HC, Jr., Walaas SI and Greengard P. DARPP-32, a dopamine- and adenosine 3':5'-monophosphate-regulated phosphoprotein enriched in dopamine-innervated brain regions. III. Immunocytochemical localization. J Neurosci. 1984; 4:111124.

4. Hemmings HC, Jr. and Greengard P. DARPP-32, a dopamine- and adenosine 3':5'-monophosphate-regulated phosphoprotein: regional, tissue, and phylogenetic distribution. J Neurosci. 1986; 6:1469-1481.

5. Meister B, Fryckstedt J, Schalling M, Cortes R, Hokfelt T, Aperia A, Hemmings HC, Jr., Nairn AC, Ehrlich $\mathrm{M}$ and Greengard P. Dopamine- and cAMP-regulated phosphoprotein (DARPP-32) and dopamine DA1 agonistsensitive $\mathrm{Na}+, \mathrm{K}+-\mathrm{ATP} a \mathrm{se}$ in renal tubule cells. Proc Natl Acad Sci U S A. 1989; 86:8068-8072.

6. Hemmings HC, Jr., Greengard P, Tung HY and Cohen P. DARPP-32, a dopamine-regulated neuronal phosphoprotein, is a potent inhibitor of protein phosphatase-1. Nature. 1984; 310:503-505.

7. Greengard P, Allen PB and Nairn AC. Beyond the dopamine receptor: the DARPP-32/protein phosphatase-1 cascade. Neuron. 1999; 23:435-447.

8. Greengard P. The neurobiology of slow synaptic transmission. Science. 2001; 294:1024-1030.

9. Svenningsson P, Nishi A, Fisone G, Girault JA, Nairn AC and Greengard P. DARPP-32: an integrator of neurotransmission. Annu Rev Pharmacol Toxicol. 2004;
44:269-296.

10. Nairn AC, Svenningsson P, Nishi A, Fisone G, Girault JA and Greengard P. The role of DARPP-32 in the actions of drugs of abuse. Neuropharmacology. 2004; 47 Suppl 1:1423.

11. Svenningsson P, Nairn AC and Greengard P. DARPP-32 mediates the actions of multiple drugs of abuse. The AAPS journal. 2005; 7:E353-360.

12. El-Rifai W, Harper JC, Cummings OW, Hyytinen ER, Frierson HF, Jr., Knuutila S and Powell SM. Consistent genetic alterations in xenografts of proximal stomach and gastro-esophageal junction adenocarcinomas. Cancer Res. 1998; 58:34-37.

13. El-Rifai W, Frierson HF, Jr., Moskaluk CA, Harper JC, Petroni GR, Bissonette EA, Jones DR, Knuutila S and Powell SM. Genetic differences between adenocarcinomas arising in Barrett's esophagus and gastric mucosa. Gastroenterology. 2001; 121:592-598.

14. El-Rifai W, Smith MF, Jr., Li G, Beckler A, Carl VS, Montgomery E, Knuutila S, Moskaluk CA, Frierson HF, Jr. and Powell SM. Gastric cancers overexpress DARPP-32 and a novel isoform, t-DARPP. Cancer Res. 2002; 62:40614064 .

15. Valjent E, Pascoli V, Svenningsson P, Paul S, Enslen H, Corvol JC, Stipanovich A, Caboche J, Lombroso PJ, Nairn AC, Greengard P, Herve D and Girault JA. Regulation of a protein phosphatase cascade allows convergent dopamine and glutamate signals to activate ERK in the striatum. Proceedings of the National Academy of Sciences of the United States of America. 2005; 102:491-496.

16. Brami-Cherrier K, Valjent E, Herve D, Darragh J, Corvol JC, Pages C, Arthur SJ, Girault JA and Caboche J. Parsing molecular and behavioral effects of cocaine in mitogen- and stress-activated protein kinase-1-deficient mice. J Neurosci. 2005; 25:11444-11454.

17. Svenningsson $\mathrm{P}$, Lindskog $\mathrm{M}$, Ledent C, Parmentier M, Greengard P, Fredholm BB and Fisone G. Regulation of the phosphorylation of the dopamine- and cAMP-regulated phosphoprotein of $32 \mathrm{kDa}$ in vivo by dopamine D1, dopamine D2, and adenosine A2A receptors. Proceedings of the National Academy of Sciences of the United States of America. 2000; 97:1856-1860.

18. Ferre S, Fredholm BB, Morelli M, Popoli P and Fuxe K. Adenosine-dopamine receptor-receptor interactions as an integrative mechanism in the basal ganglia. Trends Neurosci. 1997; 20:482-487.

19. Schiffmann $\mathrm{SN}$ and Vanderhaeghen JJ. Adenosine A2 receptors regulate the gene expression of striatopallidal and striatonigral neurons. J Neurosci. 1993; 13:1080-1087.

20. Svenningsson $\mathrm{P}$, Lindskog $\mathrm{M}$, Rognoni $\mathrm{F}$, Fredholm $\mathrm{BB}$, Greengard $\mathrm{P}$ and Fisone G. Activation of adenosine A2A and dopamine D1 receptors stimulates cyclic AMPdependent phosphorylation of DARPP-32 in distinct populations of striatal projection neurons. Neuroscience. 
1998; 84:223-228.

21. Montmayeur JP, Bausero P, Amlaiky N, Maroteaux L, Hen $\mathrm{R}$ and Borrelli E. Differential expression of the mouse D2 dopamine receptor isoforms. FEBS Lett. 1991; 278:239243.

22. Lindgren N, Usiello A, Goiny M, Haycock J, Erbs E, Greengard P, Hokfelt T, Borrelli E and Fisone G. Distinct roles of dopamine $\mathrm{D} 2 \mathrm{~L}$ and $\mathrm{D} 2 \mathrm{~S}$ receptor isoforms in the regulation of protein phosphorylation at presynaptic and postsynaptic sites. Proceedings of the National Academy of Sciences of the United States of America. 2003; 100:43054309.

23. Nishi A, Snyder GL, Fienberg AA, Fisone G, Aperia A, Nairn AC and Greengard P. Requirement for DARPP-32 in mediating effect of dopamine D2 receptor activation. Eur J Neurosci. 1999; 11:2589-2592.

24. Ouimet CC, Langley-Gullion $\mathrm{KC}$ and Greengard P. Quantitative immunocytochemistry of DARPP-32expressing neurons in the rat caudatoputamen. Brain Res. 1998; 808:8-12.

25. Yan Z, Hsieh-Wilson L, Feng J, Tomizawa K, Allen PB, Fienberg AA, Nairn AC and Greengard P. Protein phosphatase 1 modulation of neostriatal AMPA channels: regulation by DARPP-32 and spinophilin. Nat Neurosci. 1999; 2:13-17.

26. Fienberg AA, Hiroi N, Mermelstein PG, Song W, Snyder GL, Nishi A, Cheramy A, O'Callaghan JP, Miller DB, Cole DG, Corbett R, Haile CN, Cooper DC, Onn SP, Grace AA, Ouimet CC, et al. DARPP-32: regulator of the efficacy of dopaminergic neurotransmission. Science. 1998; 281:838842.

27. Bibb JA, Snyder GL, Nishi A, Yan Z, Meijer L, Fienberg AA, Tsai LH, Kwon YT, Girault JA, Czernik AJ, Huganir RL, Hemmings HC, Jr., Nairn AC and Greengard P. Phosphorylation of DARPP-32 by Cdk5 modulates dopamine signalling in neurons. Nature. 1999; 402:669671.

28. Gray PC, Johnson BD, Westenbroek RE, Hays LG, Yates JR, 3rd, Scheuer T, Catterall WA and Murphy BJ. Primary structure and function of an A kinase anchoring protein associated with calcium channels. Neuron. 1998; 20:10171026.

29. Yger M and Girault JA. DARPP-32, Jack of All Trades... Master of Which? Front Behav Neurosci. 2011; 5:56.

30. Desdouits F, Cohen D, Nairn AC, Greengard P and Girault JA. Phosphorylation of DARPP-32, a dopamine- and cAMP-regulated phosphoprotein, by casein kinase I in vitro and in vivo. The Journal of biological chemistry. 1995; 270:8772-8778.

31. Desdouits F, Siciliano JC, Nairn AC, Greengard P and Girault JA. Dephosphorylation of Ser-137 in DARPP-32 by protein phosphatases $2 \mathrm{~A}$ and $2 \mathrm{C}$ : different roles in vitro and in striatonigral neurons. The Biochemical journal. 1998; 330:211-216.
32. Girault JA, Hemmings HC, Jr., Williams KR, Nairn AC and Greengard P. Phosphorylation of DARPP-32, a dopamineand cAMP-regulated phosphoprotein, by casein kinase II. The Journal of biological chemistry. 1989; 264:2174821759.

33. Neyroz P, Desdouits F, Benfenati F, Knutson JR, Greengard $\mathrm{P}$ and Girault JA. Study of the conformation of DARPP-32, a dopamine- and cAMP-regulated phosphoprotein, by fluorescence spectroscopy. The Journal of biological chemistry. 1993; 268:24022-24031.

34. Kunii Y, Hyde TM, Ye T, Li C, Kolachana B, Dickinson D, Weinberger DR, Kleinman JE and Lipska BK. Revisiting DARPP-32 in postmortem human brain: changes in schizophrenia and bipolar disorder and genetic associations with t-DARPP-32 expression. Mol Psychiatry. 2014; 19:192-199.

35. Albert KA, Hemmings HC, Jr., Adamo AI, Potkin SG, Akbarian S, Sandman CA, Cotman CW, Bunney WE, Jr. and Greengard P. Evidence for decreased DARPP-32 in the prefrontal cortex of patients with schizophrenia. Archives of general psychiatry. 2002; 59:705-712.

36. Cho K, Cho MH, Seo JH, Peak J, Kong KH, Yoon SY and Kim DH. Calpain-mediated cleavage of DARPP-32 in Alzheimer's disease. Aging cell. 2015.

37. Knuutila S, Bjorkqvist AM, Autio K, Tarkkanen M, Wolf M, Monni O, Szymanska J, Larramendy ML, Tapper J, Pere H, El-Rifai W, Hemmer S, Wasenius VM, Vidgren $\mathrm{V}$ and Zhu Y. DNA copy number amplifications in human neoplasms: review of comparative genomic hybridization studies. Am J Pathol. 1998; 152:1107-1123.

38. Varis A, Wolf M, Monni O, Vakkari ML, Kokkola A, Moskaluk C, Frierson H, Jr., Powell SM, Knuutila S, Kallioniemi A and El-Rifai W. Targets of gene amplification and overexpression at $17 \mathrm{q}$ in gastric cancer. Cancer research. 2002; 62:2625-2629.

39. Belkhiri A, Zaika A, Pidkovka N, Knuutila S, Moskaluk C and El-Rifai W. Darpp-32: a novel antiapoptotic gene in upper gastrointestinal carcinomas. Cancer research. 2005; 65:6583-6592.

40. Christenson JL, Denny EC and Kane SE. t-Darpp overexpression in HER2-positive breast cancer confers a survival advantage in lapatinib. Oncotarget. 2015; 6:3313445. doi: 10.18632/oncotarget.5311.

41. Vangamudi B, Peng DF, Cai Q, El-Rifai W, Zheng W and Belkhiri A. t-DARPP regulates phosphatidylinositol-3kinase-dependent cell growth in breast cancer. Mol Cancer. 2010; 9:240.

42. Gu L, Waliany S and Kane SE. Darpp-32 and its truncated variant t-Darpp have antagonistic effects on breast cancer cell growth and herceptin resistance. PLoS One. 2009; 4:e6220.

43. Wang MS, Pan Y, Liu N, Guo C, Hong L and Fan D. Overexpression of DARPP-32 in colorectal adenocarcinoma. International journal of clinical practice. 
2005; 59:58-61.

44. Mukherjee K, Peng D, Brifkani Z, Belkhiri A, Pera M, Koyama T, Koehler EA, Revetta FL, Washington MK and El-Rifai W. Dopamine and cAMP regulated phosphoprotein MW $32 \mathrm{kDa}$ is overexpressed in early stages of gastric tumorigenesis. Surgery. 2010; 148:354-363.

45. Prensner JR and Chinnaiyan AM. Oncogenic gene fusions in epithelial carcinomas. Current opinion in genetics \& development. 2009; 19:82-91.

46. Mitelman F, Johansson B and Mertens F. The impact of translocations and gene fusions on cancer causation. Nat Rev Cancer. 2007; 7:233-245.

47. Yun SM, Yoon K, Lee S, Kim E, Kong SH, Choe J, Kang JM, Han TS, Kim P, Choi Y, Jho S, Yoo H, Bhak J, Yang HK and Kim SJ. PPP1R1B-STARD3 chimeric fusion transcript in human gastric cancer promotes tumorigenesis through activation of PI3K/AKT signaling. Oncogene. 2014; 33:5341-5347.

48. Zhu S, Chen Z, Katsha A, Hong J, Belkhiri A and ElRifai W. Regulation of CD44E by DARPP-32-dependent activation of SRp20 splicing factor in gastric tumorigenesis. Oncogene. 2015.

49. Christenson JL and Kane SE. Darpp-32 and t-Darpp are differentially expressed in normal and malignant mouse mammary tissue. Mol Cancer. 2014; 13:192.

50. Belkhiri A, Dar AA, Zaika A, Kelley M and El-Rifai W. t-Darpp promotes cancer cell survival by up-regulation of $\mathrm{Bcl} 2$ through Akt-dependent mechanism. Cancer research. 2008; 68:395-403.

51. Chang MJ, Zhong F, Lavik AR, Parys JB, Berridge MJ and Distelhorst CW. Feedback regulation mediated by Bcl2 and DARPP-32 regulates inositol 1,4,5-trisphosphate receptor phosphorylation and promotes cell survival. Proc Natl Acad Sci U S A. 2014; 111:1186-1191.

52. Hong L, Zhao Y, Wang J, Han Y, Guo W, Jin H, Zhai H, Bai F, Zhang X, Qiao T, Chen Z and Fan D. Reversal of multidrug resistance of adriamycin-resistant gastric adenocarcinoma cells through the up-regulation of DARPP-32. Dig Dis Sci. 2008; 53:101-107.

53. Zhu S, Belkhiri A and El-Rifai W. DARPP-32 increases interactions between epidermal growth factor receptor and ERBB3 to promote tumor resistance to gefitinib. Gastroenterology. 2011; 141:1738-1748 e1731-1732.
54. Belkhiri A, Zhu S, Chen Z, Soutto M and El-Rifai W. Resistance to TRAIL is mediated by DARPP-32 in gastric cancer. Clin Cancer Res. 2012; 18:3889-3900.

55. Belkhiri A, Dar AA, Peng DF, Razvi MH, Rinehart C, Arteaga CL and El-Rifai W. Expression of t-DARPP mediates trastuzumab resistance in breast cancer cells. Clin Cancer Res. 2008; 14:4564-4571.

56. Hamel S, Bouchard A, Ferrario C, Hassan S, AguilarMahecha A, Buchanan M, Quenneville L, Miller W and Basik M. Both t-Darpp and DARPP-32 can cause resistance to trastuzumab in breast cancer cells and are frequently expressed in primary breast cancers. Breast cancer research and treatment. 2010; 120:47-57.

57. Hong J, Katsha A, Lu P, Shyr Y, Belkhiri A and ElRifai W. Regulation of ERBB2 receptor by t-DARPP mediates trastuzumab resistance in human esophageal adenocarcinoma. Cancer research. 2012; 72:4504-4514.

58. Vangamudi B, Zhu S, Soutto M, Belkhiri A and El-Rifai W. Regulation of beta-catenin by t-DARPP in upper gastrointestinal cancer cells. Mol Cancer. 2011; 10:32.

59. Hansen C, Greengard P, Nairn AC, Andersson T and Vogel WF. Phosphorylation of DARPP-32 regulates breast cancer cell migration downstream of the receptor tyrosine kinase DDR1. Exp Cell Res. 2006; 312:4011-4018.

60. Hansen C, Howlin J, Tengholm A, Dyachok O, Vogel WF, Nairn AC, Greengard P and Andersson T. Wnt-5a-induced phosphorylation of DARPP-32 inhibits breast cancer cell migration in a CREB-dependent manner. J Biol Chem. 2009; 284:27533-27543.

61. Zhu S, Hong J, Tripathi MK, Sehdev V, Belkhiri A and El-Rifai W. Regulation of CXCR4-Mediated Invasion by DARPP-32 in Gastric Cancer Cells. Mol Cancer Res. 2013; 11:86-94.

62. Kopljar M, Patrlj L, Korolija-Marinic D, Horzic M, Cupurdija K and Bakota B. High Expression of DARPP-32 in Colorectal Cancer Is Associated With Liver Metastases and Predicts Survival for Dukes A and B Patients: Results of a Pilot Study. International surgery. 2015; 100:213-220.

63. Chen Z, Zhu S, Hong J, Soutto M, Peng D, Belkhiri A, $\mathrm{Xu} \mathrm{Z}$ and El-Rifai W. Gastric tumour-derived ANGPT2 regulation by DARPP-32 promotes angiogenesis. Gut. 2015. 